\title{
Learning Spiritual Behaviours as a Means to Reverse Harmful Epigenetic Changes Resulting from Domestic Violence
}

\author{
Maysar Sarieddine* \\ Phoenecia University, Saida, Lebanon
}

*Corresponding author: Maysar S, Phoenecia University, Saida, Lebanon, Tel: +009613333348; E-mail: maysar@gmail.com

Received: October 21, 2018; Accepted: October 31, 2018; Published: November 03, 2018

\begin{abstract}
Excessive stress can epigenetically alter an individual's DNA and affect mental health. For instance, women who have been exposed to domestic violence have been found to have psychopathological alterations in their behaviors and in their hypothalamus-pituitary-adrenal axis functioning. However, these changes are reversible, because people can change their genetic makeup by changing their thoughts and beliefs. This provides an opportunity for domestic violence survivors to acquire behavioral and cognitive practices that support healthier epigenetic modifications in the expression of genes. The use of strategies centered on spirituality has been proposed as a skill that can enhance resilience, which is the ability to adapt to stress and adversities. Similar to the epigenetic mechanisms involved in excessive stress, resilience can also alter gene expressions, which can support healthier neuropsychological functioning. The conclusion I draw from the review of recent research is that for victims of domestic violence, enhancing their spirituality through prayers, meditation, or cognitive reframing can lead to neuropsychological changes that can offset the negative psychopathological alterations that occur during excessive stress. Recommendations are that a study that frames spirituality as a buffer for stress caused by domestic violence could be significant in further illuminating the power of thoughts and beliefs in influencing our neurological functioning, and such a study or studies should be conducted with diverse populations and in a variety of settings.
\end{abstract}

Keywords: Epigenetic; Epigenetic change; Domestic violence; Neuropsychological functioning

\section{The Epigenetic Effects of Stress}

The term "epigenetic" refers to an effect or condition that arises from non-genetic influences on gene expression. Those influences can include environmental conditions, stress, a person's emotional state, or physical trauma. Specifically, these effects are heritable, but that heritability does not occur through the normal mechanisms by which genotypes are passed from one generation to the next--hence the prefix "epi-" which means "on, upon, or above."

The idea that experiences, emotions, responses, etc. can influence an individual's genetic makeup is one that has fallen in and out of favor in past decades, which is somewhat understandable in that it seems to violate accepted hereditary science. However, experiments have shown that animals that are subjected to stresses or other stimuli and respond to them will 
www.yumedtext.com | November-2018

transmit those responses to their offspring [1]. For instance, aversion to a given stimulus imparted by experimentally causing a negative event to occur when that stimulus is present, such as delivering an electric shock to a rat when it encounters a certain food, will often be transmitted to its offspring; the rat's offspring will exhibit the same aversion without ever having experienced the negative stimulus.

Epigenetic phenotype variations occur in plants as well as animals. The mechanism by which this occurs is not always well understood, but it is clear that organisms are fundamentally altered- at the DNA and RNA level-by their experiences. In particular, survival mechanisms may be transmitted to offspring if they are formulated in response to stimuli [2]. That these changes occur within a single generation strongly suggests that they are not an expression of Darwinian evolution concepts as such- that an organism that develops a way to cope with a stressor is more likely to reproduce. Rather, epigenetic variations are faster than evolution; nonetheless, they are often surprisingly complex [2].

The topic of epigenetic variation has unfortunately been clouded by pseudoscience [3]. A fairly common expression of this is the idea that one's genetics can be manipulated by mind control or other psychological techniques. Self-medication, hypnosis, sleep therapy, etc. have been touted as ways to influence one's genetic makeup and make certain (presumably positive) traits heritable. Similarly, it is often touted that immunity or greater resistance to disease can be conferred by thought training [3]. These ideas reflect an ignorance of what epigenetic variation actually is and how it works.

Epigenetics can potentially influence a host of human conditions. In the field of medicine, epigenetics can explain aging mechanisms and the body's responses to cancer, which are seen by some researchers to be closely related [4]. In the field of human psychology, epigenetics can be used to understand responses to stress, anxiety, depression, addiction, and fear. For example, conditional fears- fears that arise in response to a specific stimulus- are heritable traits [1].

This concept informs the purpose of this paper, which is to suggest that more research is needed on the epigenetic changes that occur in humans in response to stressors. Specifically, it is not well understood what the effects of those changes are on the individual's future behavior and ability to cope with stress. What little research exists has focused on the heritability of such changes; there has been no research on how a person might change stress-coping mechanisms as a result of thought and behavior alterations.

In particular, it is not well understood how an individual may heighten his/her positive responses to stress (by developing coping mechanisms) by positive epigenetic modification. Can an individual alter his/her genetic makeup by thinking? This might at first seem implausible, but after all, there is robust science suggesting that experiences do cause genetic modification, as there is otherwise no mechanism by which stimulus response traits could be heritable [5]. Stimulus responses in thinking creatures have an emotional component; the animal (or the human) crafts coping mechanisms on both a physiological and an emotional level [6].

Therefore, I recommend that research be done on whether and to what extent an emotional and cognitive therapy program could have a positive effect on an individual's genetic makeup and consequent ability to cope with a stressor, especially a recurring one. In particular, I would like to discuss how such thought modification could help the victims of domestic 
www.yumedtext.com | November-2018

violence to form a physical and mental shield against its effects. These would include the effects of past trauma as well as future traumatic events that are related.

If it is true that responses to stimuli produce epigenetic modifications (and that appears to be the case in this growing field), then it follows that a person who successfully copes with stressful stimuli will be better equipped to deal with that same type of stress in the future. This may seem like common wisdom, but it is important to draw the distinction that these are not direct cognitive modifications, as in learning to cope with stress (ors). Rather, these changes occur at the genetic level- the organism becomes better equipped to handle the negative event. Therefore, a person who learns ways to deal with domestic violence and its aftermath and effects will be better equipped to withstand it on a fundamental level. This and other life experiences create and influence behavior patterns, as explained below.

\section{Neurobiology of Behavior Patterns}

Behavior is self-reinforcing in that a person will tend to repeat a behavior as long as it solves a problem, deals with a situation, or at least creates no perceived harm [7]. The fact that an individual has performed a given behavior in the past increases the likelihood that it will be repeated, and that likelihood increases with each iteration. The brain forms stimuliaction patterns that reinforce these trends [7].

Mindfulness and meditation can speed and strengthen the formation of these neural behavior pathways [8]. These changes occur especially when the mind and body are aware of the benefits of the behavior pattern(s). Learning how to deal with psychological stress is a crucial defense mechanism, and mindfulness, meditation, and other spiritual practices can equip the brain with the resources and skills needed to do so [8].

\section{Quantum Mechanics of the Brain}

Al Amar [9] posited that the brain operates according to the principles of quantum mechanics. The state of the brain is constantly changing according to the actions and states of its billions of neurons and the electrical impulses traveling among them. Al Amar [9] mentioned the brain's "plasticity," which is its adaptational and learning abilities. This includes beliefs and emotions, which when expressed, create a feedback mechanism, which is often pleasurable. Thus, the quantum state of the brain is influenced by experiences, which include psychotherapy.

\section{The Biology of Belief: Lipton's Work}

Bruce Lipton originally published his seminal work in 2005. As a cell biologist, he initially was taught to believe what he called genetic determinism- that a person's behavior was determined (at least, to a great extent) by cellular programming, or genetics and that that fundamental aspect could not be altered [10]. Specifically, behavioral changes would not be imprinted on a person's genes and were thus not directly heritable. Lipton challenged conventional thinking, which was based on a Darwinian perspective, that behavior may be learned by an organism but that learned behavior will not cause changes at the genetic level. Yet, Lipton pointed out, human genes are switched on and off by external stimuli. Thus, a person's gene pattern is the sum of his genetics at birth and his experiences. 
www.yumedtext.com | November-2018

Lipton [10] reported being viewed as a heretic by many in the scientific community, being scorned by even his friends and colleagues. Lipton may not have helped his cause by stating that the human genetic pattern was infinitely mutable via thought and experience and thus, "perfect happiness" was attainable by all. Nonetheless, his views align with epigenetics, which as explained above, is a growing field. Lipton [11] stated that spirituality was a vital factor in epigenetic change and that such change, as it occurs at the cellular level, is fundamental.

It could be said that the jury is still out regarding Lipton's views. Certainly, they contradict much of accepted thinking in the fields of genetics. Yet, much recent research has suggested that there is value in his approach. This suggests the value of spiritual psychology in teaching the brain and body- at the cellular level- to become more resilient and resistant to stressors. Spiritual and mainstream psychology intersects in instances where spiritual behavior is proposed or used as a therapeutic device.

\section{Spiritual and Mainstream Psychology}

The primary difference between conventional, or mainstream psychology and spiritual psychology is that the former, while it may include in its treatment modalities the encouragement of the patient to engage in spiritual activities as a form of therapy, does not expressly ascribe any power or treatment mechanism to spiritual belief. In other words, it does not state that there is any direct benefit from praying or other spiritual practices other than as a calming and therapeutic effect on the patient. This is distinguished from spiritual psychology, which ascribes some intrinsic benefit to the patient from spiritual activities.

Spiritual psychology focuses on guiding the patient toward self-realization. It is not dependent on any particular creed or belief system. Rather, it is patient-focused and depends less on empirical knowledge than conventional psychology [12]. While it may be oriented toward the belief system of the practitioner or the patient, its goal is not so much to teach or affirm that belief system as to use is as a mechanism for the patient to achieve greater self-awareness [12]. That said, a patient who lives in a spiritual society may derive greater comfort and satisfaction from spiritual rather than conventional psychotherapy [12].

This is not to say that spirituality is a vague concept or cannot be measured. In fact, Ekşi and Kardaş [13] developed a scale to measure patients' spirituality. The three factors measured by the scale, which was administered to 497 psychiatric patients in Turkey, were transcendence, harmony with nature, and anomie. The researchers determined that the scores returned by the instrument (Likert-scale questions) were a valid and reliable measure of spirituality and that such scores could be used as a basis for the proper therapies [13].

Turning to spirituality to comfort a patient is as old as civilization itself; when a condition was incurable or unavoidably fatal, medical and psychological professionals would turn to prayer as a way of assisting the patient [14]. In extremity, the patient can be greatly comforted by the concept of an afterlife, as both a respite from suffering and an improvement of one's lot [14]. Thus, whether one ascribes validity to a particular belief system or not is moot; what matters is that the patient feels better as a result of the spiritual therapy. 
www.yumedtext.com | November-2018

While spiritual psychology is practiced more in non-Western cultures, it has been more mainstream in recent years. Lukoff [15] reflected on his 35 years of clinical practice in California, in which he had integrated spiritual therapy into his psychiatry practice. He reported that integrating traditional Western empirical approaches with Eastern spiritual approaches had proved beneficial to his patients. He stated that many of his patients had achieved positive results from his therapeutic approach, which in the final analysis, is all that matters [15].

Western conventional and Eastern spiritual psychology, like Western and Eastern medicine, have begun to integrate in recent years $[15,16]$. Plante stated that it was necessary for Western psychologists to improve their professional cultural competence regarding their patients' spirituality. Also, Plante [16] held open the possibility that even Western conventional practitioners should be prepared to offer spiritual therapy to their patients.

\section{The Benefits of Spiritual Psychology}

Spiritual psychology can have benefits to the patient that are not usually conferred by mainstream (or Western) practices. These include a deeper connection between the therapist and the patient [16]. Lukoff [15] reported that a spiritual approach had worked with patients for whom other approaches had failed. The difference between spiritual psychology and mainstream psychology is that the latter does not emphasize the patient's spiritual life, which may be extremely important to the patient. Thus, for certain patients, spiritual psychology is the proper and most beneficial therapeutic approach $[15,16]$.

In a way, spiritual psychology is fundamental to the field of psychology. Therapists, whether called that at the time or something else, have used spiritual psychology to comfort and heal those who were afflicted for thousands of years [14]. Addressing a patient's spiritual life and well-being may be the best therapeutic approach even with the availability of newer techniques [14]. In many societies in the past and the present, a person's inner psyche was and is considered to be his/her spirit; therefore, any meaningful psychotherapy must be spiritual in nature [12].

\section{The Psychological Effects of Domestic Violence}

The focus of this paper is on how spiritual therapy can treat the psychological effects of domestic violence. The physical effects of domestic violence are usually obvious and quite visible; however, its psychological and emotional impacts on the victim are usually at least as severe, if not more so, and are usually much longer-lasting [17]. The emotional pain a victim of domestic violence feels requires treatment and therapy just as physical wounds do, but that damage is not as readily apparent as physical trauma. As a result, much of the damage from domestic violence goes untreated and unreported ([18].

Emotional damage from being a victim of domestic violence includes heightened fear, lowered self-esteem, difficulty in forming new relationships, damage to existing relationships, and difficulties in establishing and maintaining trust. In this context, a victim is anyone who is traumatized as a result of the abuse, whether its direct target or not. This includes the children of battered spouses [19]. Thus, mitigating the problem of domestic violence must include recognizing all of its victims; some such victims may be hidden if they are not the ones assaulted by the abuser.

Persons who experience domestic violence may suffer lowered emotional intelligence as a result [20]. These researchers studied a population of 40 women in Poland who had been the victims of domestic violence. They administered an emotional 
www.yumedtext.com | November-2018

intelligence survey instrument to them and to a control group who had not experienced domestic violence. The scores on the assessment were lower for the group that had suffered domestic violence. The authors suggested that psychosocial interventions should take into account the possibility that abused persons may have had their emotional intelligence degraded as a result of the abuse, meaning that they would have less resilience, lowered emotional thresholds, and lower tolerance for further trauma [20].

Interventions for the victims of domestic violence include raising the victim's self-esteem and returning him/her to a healthy level of emotional functioning. The victim of such violence may feel, and have felt, powerless to resist the abuser and/or control his/her fate. It is problematic, however, for a therapist to counteract the emotional damage that has been done, as the victim may be reassured by hearing that he/she is a worthy human being and a release from the status of victim; but on the other hand, the victim's recent life experience is in fact that of being a victim and a target of abuse, which contradicts any positive feelings of self-esteem.

From the epigenetic viewpoint, the abuse causes alterations at the genetic level. The mind and body react to the abuse with the goal of mitigating its effects and preparing for it should it happen again. These can take a positive form (increased resilience, resolve to fight back, increased vigilance, mental and physical separation from the abuser) or a negative one (withdrawal, defensiveness, internalizing victimhood, lack of assertiveness). The goal of the therapist should be to undo the negative changes if at all possible. Epigenetic effects could be made to occur relatively quickly if the victim's thoughts and emotions can be re-aligned. This can be accomplished through the actions of trained interventionists.

\section{Interventions to Treat Domestic Violence Victims}

The first step that responders should take is to ensure the victim's safety and to put him/her in a safe space where the abuse cannot recur; this is a fundamental step that must be taken before any treatment can occur. If the violence occurs within a family unit, this step may be highly disruptive to the victim(s), as often, a new place to live must be found while the problem is dealt with by law enforcement and the courts [21]. Even if the offender is removed and the victim can stay in his/her own house, however, there will still be problems of psychological damage and trauma, such as post-traumatic stress disorder (PTSD) [21].

Depending on the metrics used, roughly $3 / 4$ of female victims of domestic violence suffer some kind of long-lasting mental trauma (i.e., it does not stop when the abuse stops) [22]. These effects include depression, PTSD, loss of self-esteem, developing substance abuse, and in some cases, self-inflicted violence [22]. Victims of domestic violence rarely emerge unscathed from the experience and in many cases, suffer permanent psychological and emotional damage [22].

PTSD is particularly prevalent in the victims of domestic abuse. In addition to the obvious effects of physical assault, the victim suffers a loss of security and sense of place, as the home is no longer a place of safety and security but rather, a place where abuse happens or has happened [23]. The fundamental nature of suffering an assault or assaults in one's home makes the resultant psychological damage that much harder to heal and to treat [23]. 
www.yumedtext.com | November-2018

Domestic violence support services are available in most medium-size and large communities. The conditions that professionals offering such services treat often include depression, anxiety, and post-traumatic stress disorder (PTSD) [24]. Therefore, the professional who encounters and treats domestic abuse victims will usually follow a fairly standard protocol in assessing the psychological damage done to the victim. This will include raising the victim's self-esteem, treating his/her depression, and insulating him/her from anxiety triggers [24]. Unfortunately, sometimes either funding or facilities (shelter), or both, are not available. The effects of domestic violence and abuse can be long-lasting, but therapy and other interventions may not be provided for a long enough time.

It is for this reason that I am recommending that a study be performed on how victims of domestic violence can be helped via a program of spirituality to enhance their resilience and thereby improve their neurophysiological functioning. It is a premise inherent in this recommendation that such a program could enhance victims' gene expressions to provide them with a stronger defense mechanism against the effects of domestic violence. This would include giving them the means to mitigate the aftereffects of past abuse as well as better cope with future incidents, which is unfortunately a needed defense when the victim has no practical choice but to return to the scene of the abuse, at least in the short term.

A program of spirituality has the potential to heal victims' psychological damage by providing them with a grounded space in which to shelter. Prayers, meditation, and cognitive reframing can all help to create such a space. This last, cognitive reframing, is a therapeutic exercise whereby the victim reframes her experience in a more positive, or at least a less negative light. This could include convincing the victim that the abuse was not his/her fault (a common perception in victims, which often leads to further psychopathology) or that its severity is serving as a "wake-up call" and the problem will now be dealt with [25]. Helping the victim to bring spirituality into his/her life can have intrinsic therapeutic value and enhance the victim's well-being [26]. This approach to therapy does not have to be aligned with any particular religion or belief system; approaches in the past have involved religious conversions or at least the attempt to do so, but that is not necessary to help and heal victims [25].

The epigenetic viewpoint suggests that if successful, spirituality therapy could alter the victim's neuropsychological makeup at the fundamental level- by altering his/her genotype. The victim would acquire greater emotional and psychological resilience. Also, the lingering effects of the abuse- such as PTSD- would lessen in severity and perhaps duration as well [27].

While it would be a practical impossibility to discover experimentally whether these therapeutic changes are heritable, it would be quite possible to evaluate victims before and after spirituality therapy. A premise of such a study, based on the epigenetic viewpoint, would be that any changes in behavior and emotions brought about by the therapy would not only be learned but also "imprinted"- that the person has the improvements conferred by the therapy encoded in his/her genotype. Again, it would be impossible to observe this effect directly, but it would be valuable to do a before-and-after comparison nonetheless.

\section{Recommendations for Future Studies}

Such a comparison could be performed using either quantitative or qualitative methods. Characteristics such as resiliency and emotional health (also negatives such as stress, trauma, and PTSD) can be measured quantitatively via Likert-scale 
www.yumedtext.com | November-2018

questionnaires; there are many existing validated instruments for that purpose and the resultant variables can be compared in a number of ways. Such approaches, however, though "neat and tidy," can only explore the specific aspects of the phenomenon of domestic abuse and recovery therefrom that are dealt with in a given instrument. In other words, such an approach is self-limiting.

A qualitative phenomenological study could uncover deep insights about the lived experiences of those who suffer domestic abuse and receive spirituality therapy to treat its effects. As the effects of any kind of psychological therapy are best discovered through self-reporting or by direct observation by the researcher, qualitative open-ended interviews have the potential to provide rich and detailed data. (Also, it would be impractical as well as ethically questionable to observe persons who have been previously traumatized, even with their permission).

Following are some approaches that could be used:

- Locate a therapy/treatment center that treats and counsels victims of domestic violence. Either locate a therapist who already uses spirituality therapy or ask a therapist to employ it, with patient/victim consent. Interview the participants (the victims who receive the therapy) both before and after receiving the spirituality therapy. Perform thematic analyses of the two interviews and compare them for each participant. The sample size here could be quite small, as few as six participants.

- Locate a facility that provides spiritual therapy- not necessarily or exclusively to domestic violence victims. Ask the facility to ask permission of any domestic violence victims who use its services to be participants in a study. This could be conducted as above insofar as procedures and sampling.

- Locate potential participants who have been victims of domestic violence and ask them if they have employed spirituality in their efforts to recover from the abuse they suffered. Perform qualitative interviews with those who reply in the affirmative. This approach would not compare "before" and "after" the therapy and would probably need a larger sample size: possibly 10-12 participants.

- Locate persons who provide spiritual therapy services and ask them what improvements they have seen in clients who came to them after having suffered domestic abuse. It might be difficult to locate very many participants in this approach, so this is possibly best for a case study approach. The researcher would have to be extremely careful to protect patient confidentiality and anonymity, but that is true for all the other approaches listed above.

\section{Benefits of the Recommended Research}

Victims of domestic violence could benefit by having access to a treatment method (spirituality therapy) that is presently not often used. By separating out spirituality treatment and training from the practices and tenets of any particular religion, the scope of and possibility for this therapy could be broadened. Even if spirituality therapy does not work for everyone, its availability as a treatment modality- validated by research- would be another treatment option for the victims of domestic abuse in addition to more "conventional" treatments.

Also, looking ahead to the long term, such research could add to the discipline of genetics by showing how epigenetic changes create and reinforce psychological tendencies for victims of stress. It is possible, even likely given the research so 
www.yumedtext.com | November-2018

far, that persons who successfully cope with stress become more resilient on a fundamental, genetic level. This would, theoretically, be manifested both in their own future behavior and in that of their offspring- a fascinating area to explore.

\section{REFERENCES}

1. Cortijo S, Wardenaar R, Colomé-Tatché M, et al. Mapping the epigenetic basis of complex traits. Science. 2014;343(6175):1145-8.

2. Schmitz RJ. The secret garden- epigenetic alleles underlie complex traits. Science. 2014;343(6175):1082-3.

3. Heard E, Martienssen RA. Transgenerational epigenetic inheritance: myths and mechanisms. Cell. 2014;157(1):95109.

4. Heyn H, Sayols S, Moutinho C, et al. Linkage of DNA methylation quantitative trait loci to human cancer risk. Cell Reports. 2014;7(2):331-8.

5. Liebers R, Rassoulzadegan M, Lyko F. Epigenetic regulation by heritable RNA. PLoS Genetics. 2014;10(4):e1004296.

6. Gapp K, Von Ziegler L, Tweedie-Cullen RY, et al. Early life epigenetic programming and transmission of stressinduced traits in mammals: how and when can environmental factors influence traits and their transgenerational inheritance? Bioessays. 2014;36(5):491-502.

7. Mullins A. The neuroscience of character development. Independence. 2015;40(1):68:70-2.

8. Esch T. The neurobiology of meditation and mindfulness. In: Stefan S, Harald W, editors. MeditationNeuroscientific Approaches and Philosophical Implications. Germany: Springer; 2014. 153-73 p.

9. Al Amar O. Quantum mechanics of the human brain and consciousness. Oxford, UK: Oxford University Press. 2016.

10. Lipton BH. The Biology of Belief 10th Anniversary Edition: Unleashing the Power of Consciousness, Matter \& Miracles. California: Hay House, Inc, USA; 2015.

11. Lipton B. Revealing the wizard behind the curtain: The new "Biology and Epigenetics." In Measuring the Immeasurable: The Scientific Case for Spirituality. Colorado: Sounds True Inc, USA; 2008. 185-200 p.

12. Oman D, Singh NN. Combining Indian and Western spiritual psychology: Applications to health and social renewal. Psychol Stud. 2018;63(2):172-80.

13. Ekşi H, Kardaş S. Spiritual well-being: Scale development and validation. Spiritual Psychol Couns. 2017;2(1):7388.

14. Hatun, O, Yavuz-Birben, F, İnce, Z, et al. The ticket to heaven: A spiritual resource for coping with disability. Spiritual Psychol Couns. 2016;1(2):209-235.

15. Lukoff, D. From personal experience to clinical practice to research: A career path leading to public policy changes in integrating spirituality into mental health. Spiritual Clin Pract. 2014;1(2):145.

16. Plante TG. Four steps to improve religious/spiritual cultural competence in professional psychology. Spiritual Clin Pract. 2014;1(4):288-92.

17. McCann L, Pearlman LA. Psychological trauma and adult survivor theory: Therapy and transformation. New York, NY: Routledge, USA; 2015.

18. Pain R. Seismologies of emotion: fear and activism during domestic violence. Soc Cult Geogr. 2014;15(2):127-50. 
19. Swanston J, Bowyer L, Vetere A. Towards a richer understanding of school-age children's experiences of domestic violence: The voices of children and their mothers. Clin Child Psychol Psychiatry. 2014;19(2):184-201.

20. Tsirigotis K, Łuczak J. Emotional intelligence of women who experience domestic violence. Psychiatric Q. 2016;87(1):165-76.

21. Straus MA, Gelles RJ, Steinmetz SK. Behind closed doors: Violence in the American family. New York: Routledge, USA; 2017.

22. Oram S, Khalifeh H, Howard LM. Violence against women and mental health. Lancet Psychiatry. 2017;4(2):159-70.

23. Karatzias T, Shevlin M, Fyvie C, et al. Evidence of distinct profiles of posttraumatic stress disorder (PTSD) and complex posttraumatic stress disorder (CPTSD) based on the new ICD-11 Trauma Questionnaire (ICD-TQ). J Affect Disord. 2017;207:181-7.

24. Ferrari G, Agnew-Davies R, Bailey, et al. Domestic violence and mental health: A cross-sectional survey of women seeking help from domestic violence support services. Glob Health Action. 2016;9(1):29890.

25. Lilly MM, Howell KH, Graham-Bermann S. World assumptions, religiosity, and PTSD in survivors of intimate partner violence. Violence Against Women. 2015;21(1):87-104.

26. Blakey JM. The role of spirituality in helping African American women with histories of trauma and substance abuse heal and recover. Soc Work Christianity. 2016;43(1):40-59.

27. Drumm R, Popescu M, Cooper L, et al. "God just brought me through it": Spiritual coping strategies for resilience among intimate partner violence survivors. Clin Soc Work J. 2014;42(4):385-94. 